\title{
Blood loses it when nerves go bad
}

\author{
Cell Research (2014) 24:1151-1152. doi:10.1038/cr.2014.98; published online 29 July 2014
}

\begin{abstract}
Myeloproliferative neoplasms are diseases that arise in the stem cells of the blood. In a recent paper published in Nature, Arranz et al. demonstrated that abrogation of sympathetic nerve fibers reduced bone marrow Nestin ${ }^{+}$ mesenchymal cells, which in turn led to an expansion of hematopoietic stem cells and progression of myeloproliferative neoplasms.
\end{abstract}

The stromal cell compartment, or non-hematopoietic cells, of the bone marrow has emerged as an important driver of cell state in hematopoietic stem cells (HSCs) and hematopoietic stem/progenitor cells (HSPCs) in a nonautonomous manner. The HSC niche is not well defined and a number of studies suggest that there are specialized niches for the unique regulation of HSCs and HSPCs [1]. Several studies suggest that HSCs reside in a perivascular niche in which a heterogenic population of perivascular mesenchymal stromal cells (MSCs) with overlapping expression of Nestin, LepR, Prx1, or Mxl each synthesize multiple factors (e.g., CXCL12 and $\mathrm{SCF}$ ) that promote maintenance and/or localization of HSCs $[1,2]$. Endothelial cells $\left(\mathrm{Tie}^{+}\right)$[3], and $\mathrm{MSCs}\left(\mathrm{NG2}^{+} /\right.$ $L e p R^{-}$) surrounding arterial vessels [4], are other units of the niche reported to regulate HSC numbers and quiescence, respectively. Furthermore, osteoprogenitors lining the endosteal surface have a more indirect role in the regulation of HSCs [6]. Thus, it appears that hematopoietic regulation and differentiation in the bone marrow microenvironment is governed at multiple levels [5].

Increasing evidence suggests that dysregulation of the bone marrow microenvironment may participate in blood malignancies. For instance, perturbations of the miRNA processing or ribosomal components through Dicer1 deletion in immature osteolineage cells induced myelodysplasia in mice, followed by the rare emergence of acute myeloid leukemia (AML) [6]. Others reported that $\beta$-catenin stabilization in mature osteolineage cells resulted in Notch pathway activation, myelodysplastic syndrome, and highly penetrant AML in mice [7]. In humans, $\sim 5 \%$ of post-transplant AML patients relapse with a leukemia of donor cell origin, suggesting that some patients may have a microenvironmental driver of leukemogenesis [8]. Together, these studies are consistent with a role of the bone marrow microenvironment in maintaining the integrity of hematopoiesis and restricting oncogenesis. When the well-orchestrated regulation of hematopoiesis is disrupted, blood malignancies might occur.

The study by Arranz et al. [9] is a continuation of prior work identifying perivascular bone marrow Nestin ${ }^{+}$ MSCs affected by sympathetic nerve fibers to regulate HSCs [10]. Previous studies that a perturbed bone marrow microenvironment modulates myeloproliferative neoplasms (MPNs) $[6,7]$ prompted the authors to further investigate the role of Nestin ${ }^{+}$MSCs in MPN, specifically MPN associated with Janus kinase 2 (JAK2) mutations [11, 12].

The authors first analyzed Nestin expression in bone marrow samples from MPN patients and discovered that despite elevated blood-vessel density, $\mathrm{Nestin}^{+}$cell numbers and mRNA expression were reduced. Similar findings were observed in genetically engineered mice that recapitulate human MPNs (e.g., Mx1-cre; JAK2 $2^{V 617 F}$ ), indicating that Nestin ${ }^{+}$MSCs might play a role in MPN. Arranz et al. proceeded to investigate whether a selective depletion of Nestin ${ }^{+}$MSCs mimics the MPN mouse model. Mice depleted of Nestin ${ }^{+}$ MSCs showed an expansion of HSCs, due to a drop in CXCL12 expression, accompanied by increased hematopoietic progenitors in bone marrow, peripheral blood and spleen, indicative of MPN. Extensive genome-wide RNAsequencing studies revealed enrichment of Schwann cell- and neural-related genes in Nestin ${ }^{+}$MSCs derived from MPN mice. This result prompted the authors to explore the role of sympathetic nerve fibers and nonmyelinating Schwann cells in MPN patients and the MPN mouse model. Strikingly, both MPN patients and MPN mice had reduced sympathetic nerve fibers and nonmyelinating Schwann cells adjacent to Nestin ${ }^{+}$cells in the bone marrow. Multiplex ELISA experiments identified that mutant HSCs secrete IL-1 $\beta$, which induced apoptosis in bone marrow Schwann cells by Caspase- 1 activation followed by neuronal damage. Neuralglial damage in turn compromised $\mathrm{Nestin}^{+}$MSCs survival and led to MPN. Finally, the authors rescued the MPN phenotype partially in MPN mice by treating the mutant mice with IL-1R antagonist, a neuroglial protection agent (4-methylcatechol), or a $\beta 3$-adrenergic agonist (BRL37344) which compensated for deficient sympathetic stimulation. This treatment was selective against mutant hematopoietic progenitors and preserved normal HSCs, and this effect could only be observed in vivo. There- 


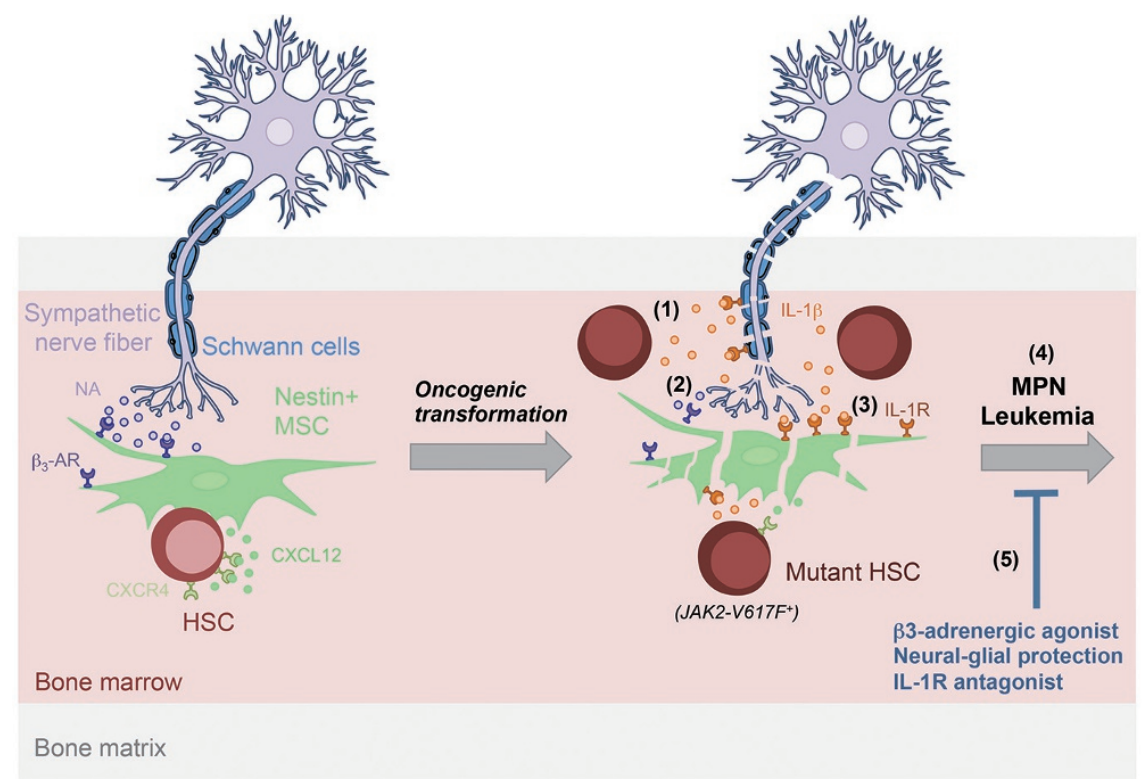

Figure 1 Bone marrow neuropathy leads to mutant HSC expansion in MPN. (1) IL$1 \beta$ is released by mutant HSCs, which induces Caspase-1-dependent apoptosis in sympathetic nerve fibers, ensheathed by nonmyelinating Schwann cells. (2) Neuralglial damage leads to a reduced noradrenergic sympathetic stimulation of Nestin ${ }^{+}$ MSCs and loss of MSCs. (3) Aberrant neural regulation sensitizes Nestin ${ }^{+}$MSCs to IL-1 $\beta$-induced apoptosis with a subsequent drop in CXCL12 expression. (4) HSC and progenitor cell proliferation is increased, followed by MPN pathogenesis. (5) $\mathrm{Nestin}^{+}$MSCs survival and function can be restored by the neuroglial protective agent 4-methylcatechol, the $\beta 3$-adrenergic agonist BRL37344, or by blocking IL-1R. MPN, myeloproliferative neoplasm; HSC, hematopoietic stem cell; MSC, mesenchymal stem cell; NA, noradrenaline; AR, adrenergic receptor; IL, Interleukin; IL-1R, Interleukin-1 receptor.

fore, the authors concluded that the effect was niche-dependent (Figure 1).

While the prevailing understanding of cancer as a disease in which changes in the cell of origin drive oncogenic transformation, these studies point to the potential for the microenvironment as a critical cooperator in the malignant process for at least some neoplasms. This study emphasizes that there may required for $\mathrm{MPN}$. HSCs acquire a mutation (e.g., JAK2-V617F mutation) that leads to cell expansion and the mutant HSC perturbs the bone marrow niche, which further drive HSCs into neoplasia. Based on these findings, the authors postulated that neural-glial protective agents and $\beta 3$-adrenergic agonists may subvert the process and be therapeutically useful. Therefore, this model provides insight into how the neural compartment of the bone marrow microenvironment can serve as a modulator of malignancy and offers a novel, testable approach for treating MPNs - by not only targeting the malignant cell, but also by selectively targeting the unhealthy niche.

Ninib Baryawno ${ }^{1,2,3}$,
David T Scadden $^{1,2,3}$

${ }^{I}$ Center for Regenerative Medicine, Massachusetts General Hospital, Boston, MA 02114, USA; ${ }^{2}$ Department of Stem Cell and Regenerative Biology, Harvard University, Cambridge, MA 02138, USA; ${ }^{3}$ Harvard Stem Cell Institute, Cambridge, MA 02138, USA

Correspondence: David T Scadden

E-mail: david_scadden@harvard.edu

\section{References}

1 Morrison SJ, Scadden DT. Nature 2014; 505:327-334.

2 Zhou BO, Yue R, Murphy MM, et al. Cell Stem Cell 2014; 15:154-168.

3 Ding L, Saunders TL, Enikolopov G, Morrison SJ. Nature 2012; 481:457-462.

4 Kunisaki Y, Bruns I, Scheiermann C, et al. Nature 2013; 502:637-643.

5 Scadden DT. Cell 2014; 157:41-50.

6 Raaijmakers MH, Mukherjee S, Guo S, et al. Nature 2010; 464:852-857.

7 Kode A, Manavalan JS, Mosialou I, et al. Nature 2014; 506:240-244.
8 Wiseman DH. Biol Blood Marrow Transplant 2011; 17:771-789.

9 Arranz L, Sánchez-Aguilera A, Martín-Pérez D, et al. Nature 2014; 512:78-81.

10 Mendez-Ferrer S, Michurina TV, Ferraro F, et al. Nature 2010; 466:829-834.

11 James C, Ugo V, Le Couedic JP, et al. Nature 2005; 434:1144-1148.

12 Kralovics R, Passamonti F, Buser AS, et al. $N$ Engl J Med 2005; 352:1779-1790. 